\title{
La cultura y subculturas en las instituciones. Su importancia en los procesos por la calidad y el cambio en la educación superior
}

Aida B. Azze Pavón.

Recibido: 15 abril 2013

Aceptado: 05 junio 2013

\section{RESUMEN}

Sin pretensiones de constituirse en un trabajo científico, se presenta un conjunto de consideraciones en el ámbito de los procesos autoevaluación y acreditación de los programas o carreras, orientados al mejoramiento de la calidad en Educación Superior, en el que la cultura a nivel institucional y de cada una de sus áreas académicas y administrativas toma especial importancia El sustento teórico de estas reflexiones, ha sido los temas desarrollados y las capacidades adquiridas en el curso internacional "Gestión de la Calidad y el Cambio en Educación Superior" conocido como Unicambio XXI, al que asistimos en la promoción 2003-2005, en el que compartimos experiencias y vivencias de Universidades Alemanas y Centroamericanas, aprendizajes puestos en práctica a partir del año 2005, en los procesos por la calidad compartidos con las carreras de la Escuela de Ciencias Exactas y Naturales.

Palabras claves: Cultura, cultura institucional, subculturas, universidades, dimensión humana, calidad, procesos de cambio.

\section{ABSTRACT}

Unpretentious by the author of becoming a scientific work. We present a set of considerations in the field of self-evaluation processes and accreditation of programs or careers, aimed at improving the quality of Higher Education, which at the institutional culture and each of its academic areas administrative and takes special importance; The theoretical basis of these considerations, has been developed topics and skills acquired in the international course "Quality Management and Change in Higher Education" known as UniCambio XXI, which assist in the promotion 2003-2005, in which share experiences and life of German and Central American Universities, implemented learnings from the year 2005, in the processes for quality careers shared with the School of Exacts and Natural Sciences.

Keys words: ulture, institutional culture, subcultures, universities, human dimension, quality, change processes.

\section{Introducción}

Cultura...." Aquel todo complejo que incluye el conocimiento, las creencias, el arte, la moral, el derecho, las costumbres y cualquiera de otros hábitos y capacidades adquiridos por el hombre. La situación de la cultura en las diversas sociedades de la especie humana, en la medida que puedes ser investigada según principios generales, es un objeto apto para el estudio de las leyes del pensamiento y la acción del hombre. Tylor (1995)

La cultura a su vez es un elemento cambiante y dinámico que responde a las realidades de sus individuos y grupos sociales que los habitan. Impacta y es impactada por la sociedad que la vive, pero no es la cultura representada únicamente por individuos o grupos sociales, (vistos estos de manera antropológica o sociológica). La cultura puede y de hecho, es parte integrante de las organizaciones económicas, comerciales e institucionales y responde a sus interioridades de la misma forma que actúa personal o grupalmente.

Las universidades como instituciones que aglutinan individuos, no quedan ajenas a estos supuestos. La vida de las instituciones académica, está marcada y es cambiada continuamente por su realidad cultural. Esta cultura, llamada por unos, cultura institucional, por otros cultura organizacional, es entonces, indudablemente un elemento básico a considerar al desarrollar procesos de calidad y el cambio en las instituciones de educación superior. 
"Las universidades, facultades y departamentos, hasta las disciplinas científicas mismas tienen sus propias culturas. Y son las culturas las quemuchas veces inconscientemente-deciden sobre las acciones. Las culturas, con sus diferencias, inciden clandestinamente en los detalles de la labor diaria universitaria”. (Wesseler,M. 2004)

Esta relación directa entre la cultura institucional y el desarrollo de los procesos por la calidad de cada escuela y sus carreras o programas, nos ha permitido en la práctica enfocar la visión de los procesos de la calidad y el cambio, atendiendo a las particularidades culturales de las escuelas, carreras y profesiones.

\section{La dimensión humana en los procesos por la calidad y el cambio}

El estudio de numerosas definiciones del concepto cultura institucional nos ha permitido resumirlas "como una realidad, en que se comparten acciones diarias, normas y valores, creencias e ideología, compartida y aprehendida individual y colectivamente, determinando y explicando el comportamiento de sus miembros, reconstruyéndose en sus propias acciones"

Desde una perspectiva de cambio es fundamental tener en cuenta la dimensión humana. Cada vez son más los expertos que insisten en la necesidad de integrar la dimensión humana en la estrategia de desarrollo, al aceptar que el comportamiento de las personas que trabajan en la organización constituye el elemento más delicado en la fase de cambio.

El estudio del componente humano de la organización es fundamental, ante cualquier redefinición de la estrategia, conviene analizar si la organización posee las aptitudes humanas en calidad y cantidad suficientes para hacerlo, si no es así, analizar en que condiciones o en que lapsus de tiempo se piensa que las podría tener.

Las instituciones de educación superior en su desarrollo requieren de asentamiento y consolidación de dimensiones técnicas de la gestión, es decir: estructuras, procesos, tecnologías, recursos tangibles y condiciones, entre otros, con el fin de garantizar a los recursos humanos la seguridad, consistencia, permanencia, en la labor que desempeñan promoviendo la sostenibilidad en la gestión por la calidad y el cambio.

En los procesos por la calidad y el cambio, debemos de tener en cuenta la cultura y las subculturas de los diferentes sectores y grupos, por su potencial decisivo, aunque muchas veces invisible a primera vista. Por lo que para poder analizar y entender una institución o una carrera, nunca será suficiente basarse en estándares internacionales, debemos de tener claro que debemos ubicarnos en el "contexto" específico con sus historias, normas y valores, creencias e ideología. En nuestro caso, la Universidad Estatal a Distancia (UNED) en el contexto nacional costarricense de la educación superior, con su característica única de modalidad de educación estatal a distancia y los sub-contextos de las carreras que se autoevalúan para lograr la calidad y el cambio, son algunos de los elementos que deberán tenerse en cuenta.

\section{La cultura y las subculturas en las instituciones de educación superior}

Las teorías e interpretaciones de la praxis, y los criterios e indicadores, serán más objetivos y acordes a las realidades, si se tiene en cuenta los contextos específicos, por lo que para desarrollar procesos efectivos por la calidad es importante percibir y entender la cultura específica del contexto para proceder en la forma más adecuada.

Es importante comprender que cada dependencia administrativa, académica, escuela, departamento, cátedras o disciplinas científicas, en una misma universidad tienen su propia subcultura, coexistiendo varias de ellas en el logro de un mismo fin.

Un ejemplo generalizado de diferencias culturales en las dependencias universitarias, son evidentes entre la parte académica de una universidad y la parte administrativa de la misma. En la mayoría de las universidades son evidentes las múltiples tensiones entre estos dos sectores de la universidad, que muchas veces conducen a batallas clandestinas, por la simple falta de comunicación y por un desprecio mutuo. No obstante, sabemos también que necesitamos las dos 
dimensiones para un desarrollo de la calidad. No se trata de una contradicción cultural, sino de una necesidad de interrelacionar, coordinar y comunicar la diversidad de una forma productiva.

En la UNED, he podido identificar la diferencia cultural en las Escuelas y en particular en sus carreras, debido a las características profesionales y las diferentes formas de abordar los mismos problemas y sus soluciones. En específico en la Escuela de Ciencias Exactas y Naturales, por la diversidad de carreras, se destaca que cada una de ellas tiene formas muy diferentes al organizarse y desarrollar los procesos de autoevaluación y acreditación en aras de la calidad y necesidades de cambio, sin embargo, a pesar de las diferencias, se promueve entre las carreras la cooperación y la comunicación, por ser ambos factores decisivos para la gestión de la calidad y del cambio por tratarse de retos que combinan sectores universitarios de culturas diversas.

\section{Aspectos a destacar en la cultura institucional de la UNED que favorecen los procesos de cambio}

Los conjuntos de saberes, creencias y pautas de conducta de un grupo social, incluyendo los medios materiales y las tecnologías que usan sus miembros para comunicarse entre sí y resolver sus necesidades de todo tipo, constituyen en las instituciones universitarias, manifestaciones de la cultura.

Del análisis de la Misión y Visión de la UNED, así como de los Factores de éxitos, de los Lineamientos de Política institucional 20012006 y de las Mociones del segundo Congreso Universitario, se evidencian como características de la Cultura Institucional ( UNED. 2006):

- Educación para todos. autoaprendizaje, interactividad,

- Calidad, pertinencia y equidad de la oferta académica

- Integración docencia-investigación-extensión

- Democratización del conocimiento: formación humanística, crítica, creativa.

- Compromiso con la humanidad en general y el medio ambiente.
- Presencia en las comunidades.

- Calidad en la prestación de servicios.

- Desarrollo y difusión del arte, la cultura y los derechos humanos.

- Capacitación de los funcionarios.

- Planificación estratégica.

- Autonomía de gestión en las escuelas.

- Autoevaluación y mejoramiento continúo de los programas de las carreras.

En particular la Moción 31 del 2do. Congreso Universitario de la UNED, se introducen compromisos de interés, que enriquecen los procesos de gestión de la calidad y el cambio, y que debe continuar trabajándose para consolidar la cultura institucional como son el compromiso e involucramiento de todos, el trabajo en equipos, participación, comunicación y relaciones de coordinación sobre las jerárquica, entre otros. (UNED 2006)

\section{Los retos de los cambios de la cultura institucional}

En la actualidad muchos cambios institucionales ocurren por fuerzas exteriores sobre las cuales la universidad tiene poca o ninguna influencia, como por ejemplo las tendencias internacionales y nacionales, los cambios tecnológicos como Internet o transformaciones del mercado de empleo después de un tratado de libre comercio entre naciones. A corto plazo la universidad podría defenderse, adaptarse o tratar de modificar los impactos concretos, pero a la larga no podrá descuidarlos ya que de una forma u otra tendrán efectos en la cultura institucional.

Para poder llegar más allá de una actitud de pura reacción frente a los cambios externos, la universidad puede enfocar sus esfuerzos en transformar la propia cultura proactivamente, hacia un espacio con mayor libertad de acción en sus intentos de gestión de la calidad.

El personal universitario en general puede contribuir al desarrollo de una cultura proactiva, comprendiendo la cultura existente y la necesidad de cambios, compartiendo lo nuevo y abriendo espacios para establecer nueva identidad cultural. 
Especialmente, el respeto frente a diversidades culturales hacia el interior de una universidad o también de una escuela y la confianza en una evolución dinámica de la propia institución, contribuyen a nuevas visiones compartidas y a sobrevenir resistencias. Esto vale también para las relaciones personales, la confianza en sí mismo y en la buena voluntad del otro, combinada con respeto hacia actitudes diferentes, son poderosos agentes del cambio en los equipos de trabajo.

\section{Conclusión}

En los procesos de mejora de la calidad en la educación superior, no ha de haber una nueva calidad con responsabilidad sin una atención a su cultura institucional, en relación tanto al macro ambiente nacional, como a sus particularidades reflejadas en sus características organizativas: áreas, grupos o individuos.

\section{Recomendación}

La dinámica del cambio cultural, no es un lujo para las organizaciones, es una necesidad para la sostenibilidad, pertinencia, y actualización de los procesos académicos con calidad.

\section{Referencias}

Tylor, Edward B. (1995) [1871]: "La ciencia de la cultura". En: Kahn, J. S. (comp.)

UNED (2006). Directrices de la UNED. Editorial EUNED. San José Costa Rica.

Wesseler, M. (2004) Apuntes de las conferencias brindadas en el curso Internacional UniCambio 21. Ciudad de Leipzig.

Wesseler, M. (2004) La confianza importa: como la cultura institucional puede favorecer la gestión de la calidad y el cambio en la educación superior. UniCambio 21, Modulo 2, Ciudad Panamá.

\section{Bibliografía Consultada}

Gerlach (2004) Dinámica Institucional en el context de la gestión de la calidad y el cambio: liderazgo, gestión y comunicación. UniCambio 21, Modulo 2, Ciudad Panamá.

Ruben, B. (2004). Pursuing Excellence in Higher education". Eight Fundamental Challenges, Primera edición Jossey Bass. Impreso en WILEY_USA.

Wesseler, Ramírez y Alfaro (2007) Promover la Universidad. Cinco estrategias y un dilema. Unicambio XXI. Primera Edición. Editorial EUNED. Costa Rica. 